\title{
CONVERGENCE RESULTS FOR PSEUDOSPECTRAL APPROXIMATIONS OF HYPERBOLIC SYSTEMS BY A PENALTY-TYPE BOUNDARY TREATMENT
}

\author{
DANIELE FUNARO AND DAVID GOTTLIEB
}

\begin{abstract}
In a previous paper we have presented a new method of imposing boundary conditions in the pseudospectral Chebyshev approximation of a scalar hyperbolic equation. The novel idea of the new method is to collocate the equation at the boundary points as well as in the inner grid points, using the boundary conditions as penalty terms. In this paper we extend the above boundary treatment to the case of pseudospectral approximations to general constant-coefficient hyperbolic systems of equations, and we provide error estimates for the pseudospectral Legendre method. The same scheme can be implemented also in the general (even nonlinear) case.
\end{abstract}

\section{INTRODUCTION}

The importance of the treatment of boundary conditions, in the approximation of hyperbolic systems of equations, is widely recognized. The pioneering works of Gustafsson, Kreiss, and Sundström [6] and Osher [7] provide the stability theory for the implementation of boundary conditions in the framework of the finite difference method. In fact, the question of checking the stability of a difference scheme for initial-boundary value hyperbolic problems is reduced to an algebraic problem.

The role of boundary conditions in spectral (or pseudospectral) methods is even more crucial. Spectral methods, being global in nature, are more sensitive to local treatments, such as boundary conditions, and therefore greater care must be taken in handling the boundaries.

In [3] the authors introduced a new method of applying the boundary conditions in the pseudospectral Chebyshev approximation of a scalar hyperbolic equation. The novel idea is to collocate the differential equation not only at the inner grid points, but also at the boundary itself, making use of the fact that spectral derivatives can be obtained at all grid points including the boundaries. Thus, the boundary conditions are introduced as penalty terms. In [3]

Received April 27, 1989; revised March 29, 1990.

1980 Mathematics Subject Classification (1985 Revision). Primary 65N35, 65M15.

Key words and phrases. Spectral approximation, boundary conditions, error estimates.

The second author has been sponsored by AFOSR Grant 90-0093 and by NSF Grant DMS 88-10150. 
the stability of the scheme is shown for the scalar case, and the merits of the new treatment are discussed.

The purpose of this paper is to extend the ideas presented in [3] to the case of hyperbolic systems of equations. We show how to implement the scheme for a general collocation method based on orthogonal polynomials. We also prove stability and show error estimates for the pseudospectral Legendre method applied to constant-coefficient systems. The extension of this idea to the Chebyshev method is trivial; however, it is more difficult to obtain similar convergence estimates.

The paper is organized as follows. In $\S 2$ we describe the method in the scalar case and prove error estimates for the pseudospectral Legendre method. In $\S 3$ we treat the case of the Legendre method applied to a system in diagonal form, where the unknowns are coupled via the boundary conditions. We derive error estimates for the constant-coefficient problem. In $\S 4$, we show how to implement the method for systems in nondiagonal form.

\section{ESTIMATES FOR THE SCALAR EQUATION}

In this section we derive error estimates for the new pseudospectral Legendre approximation to a scalar hyperbolic constant-coefficient problem. Let $U \equiv$ $U(x, t)$ be the solution of

$$
\begin{cases}\text { (a) } U_{t}=a U_{x}, & x \in[-1,1], 0<t<T, \\ \text { (b) } U(1, t)=h(t), & 0<t<T, \\ \text { (c) } U(x, 0)=f(x), & x \in[-1,1],\end{cases}
$$

where $h$ and $f$ are given boundary and initial functions and $a \in \mathbf{R}, a>0$.

Let $\mathbf{P}_{N}$ denote the space of algebraic polynomials of degree less than or equal to $N$. In the pseudospectral Legendre method we approximate $U$ by $v \equiv v(x, t)$, which is a $\mathbf{P}_{N}$-polynomial in the variable $x$ for any $0 \leq t<T$. This is done by demanding that $v$ satisfies equation (2.1)(a) at the grid points $x_{j}(j=1, \ldots, N)$. The points $x_{0}=1>x_{1}>\cdots>x_{N-1}>x_{N}=-1$, taken here in decreasing order, are the nodes of the Gauss-Lobatto quadrature formula, i.e., $x_{j}(j=0, \ldots, N)$ are the extrema in $[-1,1]$ of the $N$ th-degree Legendre polynomial $P_{N}$.

The choice of this particular grid allows an accurate evaluation of integrals by summing over the grid values. Namely, let $\omega_{j}(j=0, \ldots, N)$ be the weights of the Gauss-Lobatto formula; then for any polynomial $p$ of degree at most $2 N-1$ the following equality holds (see, for instance, [2]):

$$
\int_{-1}^{1} p d x=\sum_{j=0}^{N} p\left(x_{j}\right) \omega_{j} .
$$

In the following, we will set $\omega=\omega_{0}=\omega_{N}=2 / N(N+1)$.

Our new method differs from the classical one by the treatment of the boundary condition. The approximating polynomial $v$ does not satisfy exactly the 
boundary condition $(2.1)$ (b); instead, it satisfies the differential equation $(2.1)(a)$ at all the grid points with a penalty-type correction at the boundary. That is, $v$ is a solution of

$$
\left\{\begin{array}{l}
\text { (a) } v_{t}=a v_{x} \quad \text { at } x=x_{j}, j=1, \ldots, N, \\
\text { (b) } v_{t}(1, t)=a v_{x}(1, t)-\gamma(v(1, t)-h(t)), \\
\text { (c) } v\left(x_{j}, 0\right)=f\left(x_{j}\right), \quad j=0, \ldots, N .
\end{array}\right.
$$

The coefficient $\gamma \equiv \gamma_{N}>0$ in (2.3)(b) can be viewed as the amplitude of the boundary penalty term and will be specified later so that the stability of $(2.3)$ is guaranteed.

It is convenient to compare $v(\cdot, t)$ with some $\mathbf{P}_{N}$-projection of the solution $U(\cdot, t)$. To this end, we introduce two projection operators, denoted by $I_{N}$ and $\Pi_{N}$.

Definition 2.1. $I_{N} U$ is the polynomial in $\mathbf{P}_{N}$ that interpolates $U$ at the points $x_{j}(j=0, \ldots, N)$, i.e., $I_{N} U\left(x_{j}\right)=U\left(x_{j}\right), j=0, \ldots, N$.

Definition 2.2. $\Pi_{N} U$ is the polynomial in $\mathbf{P}_{N}$ that is the best approximation of $U$ in the $H^{1}(-1,1)$ norm satisfying the condition $\Pi_{N} U( \pm 1)=U( \pm 1)$.

Here, $H^{\sigma}(-1,1), \sigma \in \mathbf{R}$, is the usual Sobolev space with $H^{0}(-1,1)=$ $L^{2}(-1,1)$.

In the next theorem we estimate the error $E=\Pi_{N} U-v$. We note that, as in the Chebyshev case (see [3]), $\gamma$ has to be proportional to $N^{2}$ for stability.

Theorem 2.1. Consider the scalar equation (2.1), and let $v$ denote its pseudospectral approximation (2.3) depending on the boundary penalty amplitude $\gamma$. Then, if $\gamma \geq a / \omega=\frac{1}{2} N(N+1) a$, we have the error estimate

$$
\begin{aligned}
& \sum_{j=0}^{N} E^{2}\left(x_{j}, T\right) \omega_{j}+a \int_{0}^{T}\left(E^{2}(1, t)+E^{2}(-1, t)\right) d t \\
& \quad \leq \exp (1)\left(\sum_{j=0}^{N}\left(I_{N} f-\Pi_{N} f\right)^{2}\left(x_{j}\right) \omega_{j}+T \int_{j}^{T} \sum_{j=0}^{N} Q^{2}\left(x_{j}, t\right) \omega_{j} d t\right),
\end{aligned}
$$

where $Q=a\left[\Pi_{N} U_{x}-\left(\Pi_{N} U\right)_{x}\right]$.

Proof. Applying $\Pi_{N}$ to (2.1), one gets

$$
\begin{cases}\left(\Pi_{N} U\right)_{t}=a\left(\Pi_{N} U\right)_{x}+Q & \text { at } x=x_{j}, j=0, \ldots, N, \\ \left(\Pi_{N} U\right)(1, t)=h(t), & 0<t<T, \\ \left(\Pi_{N} U\right)\left(x_{j}, 0\right)=\left(\Pi_{N} f\right)\left(x_{j}\right), & j=0, \ldots, N .\end{cases}
$$

Therefore, upon subtracting (2.3) from (2.5), we have the following error equation:

$$
\left\{\begin{array}{l}
\text { (a) } E_{t}=a E_{x}+Q \text { at } x=x_{j}, j=1, \ldots, N, \\
\text { (b) } E_{t}(1, t)=a E_{x}(1, t)+Q(1, t)-\gamma E(1, t), \\
\text { (c) } E\left(x_{j}, 0\right)=\left(\Pi_{N} f-I_{N} f\right)\left(x_{j}\right), \quad j=0, \ldots, N .
\end{array}\right.
$$


Note that $E$ is a polynomial of degree at most $N$. Multiplying (2.6)(a) by $E\left(x_{j}, t\right) \omega_{j} \quad(j=1, \ldots, N),(2.6)(\mathrm{b})$ by $E(1, t) \omega_{0}$, and summing, we get (using (2.2)):

$$
\begin{aligned}
\sum_{j=0}^{N}\left(E_{t} E\right)\left(x_{j}, t\right) \omega_{j}= & a \int_{-1}^{1} E_{x} E d x+\sum_{j=0}^{N}(Q E)\left(x_{j}, t\right) \omega_{j} \\
& -\gamma \omega E^{2}(1, t), \quad 0 \leq t<T .
\end{aligned}
$$

Integration by parts yields

$$
\begin{aligned}
\frac{d}{d t} \sum_{j=0}^{N} E^{2}\left(x_{j}, t\right) \omega_{j}= & (a-2 \gamma \omega) E^{2}(1, t)-a E^{2}(-1, t) \\
& +2 \sum_{j=0}^{N}(Q E)\left(x_{j}, t\right) \omega_{j} \\
\leq & -a\left(E^{2}(1, t)+E^{2}(-1, t)\right)+\frac{1}{T} \sum_{j=0}^{N} E^{2}\left(x_{j}, t\right) \omega_{j} \\
& +T \sum_{j=0}^{N} Q^{2}\left(x_{j}, t\right) \omega_{j}, \quad 0 \leq t<T,
\end{aligned}
$$

where we used the assumption $a-2 \gamma \omega \leq-a$. Finally the Gronwall lemma yields (2.4).

Recall now that there exist two positive constants $C_{1}$ and $C_{2}$ such that one has, for any $p \in \mathbf{P}_{N}$ (see $[1$, p. 286]):

$$
C_{1} \int_{-1}^{1} p^{2} d x \leq \sum_{j=0}^{N} p^{2}\left(x_{j}\right) \omega_{j} \leq C_{2} \int_{-1}^{1} p^{2} d x .
$$

In addition, the following error estimates concerning the projectors $I_{N}$ and $\Pi_{N}$ can be found in [1, pp. 293 and 291]:

$$
\begin{aligned}
& \left\|U-I_{N} U\right\|_{H^{\mu}(-1,1)} \leq C N^{2 \mu-\sigma}\|U\|_{H^{\sigma}(-1,1)}, \\
& \quad \forall U \in H^{\sigma}(-1,1), \sigma>\frac{1}{2}, 0 \leq \mu \leq \sigma ; \\
& \left\|U-\Pi_{N} U\right\|_{H^{\mu}(-1,1)} \leq C N^{\mu-\sigma}\|U\|_{H^{\sigma}(-1,1)}, \\
& \quad \forall U \in H^{\sigma}(-1,1), \sigma \geq 1,0 \leq \mu \leq \sigma .
\end{aligned}
$$

Using the above inequalities, we can get an estimate of the error between the solution $U$ and the new approximate $v$.

Theorem 2.2. Let $U \in L^{2}\left(0, T ; H^{\sigma}(-1,1)\right) \cap L^{\infty}\left(0, T ; H^{\sigma-1}(-1,1)\right), \sigma \geq 1$, be the solution of (2.1). Let $v$ be the solution of the pseudospectral scheme (2.3) 
with $\gamma \geq a / \omega$. Then we have the error bounds

$$
\begin{aligned}
& \|(U-v)(\cdot, T)\|_{L^{2}(-1,1)} \\
& \quad \leq C N^{1-\sigma}\left(\sqrt{T}\|U\|_{L^{2}\left(0, T ; H^{\sigma}(-1,1)\right)}+\|U(\cdot, T)\|_{H^{\sigma-1}(-1,1)}\right. \\
& \left.+\|f\|_{H^{\sigma-1}(-1,1)}\right) \\
& \quad\left(\int_{0}^{T}(U-v)^{2}( \pm 1, t) d t\right)^{1 / 2} \\
& \quad \leq C N^{1-\sigma}\left(\sqrt{T}\|U\|_{L^{2}\left(0, T ; H^{\sigma}(-1,1)\right)}+\|f\|_{H^{\sigma-1}(-1,1)}\right) .
\end{aligned}
$$

Proof. We begin by noting that (2.11) implies for $Q=a\left[\Pi_{N} U_{x}^{\bullet}-\left(\Pi_{N} U\right)_{x}\right]$

$$
\begin{aligned}
\|Q\|_{L^{2}(-1,1)} & \leq a\left\|\Pi_{N} U-U\right\|_{H^{1}(-1,1)}+a\left\|\Pi_{N} U_{x}-U_{x}\right\|_{L^{2}(-1,1)} \\
& \leq C N^{1-\sigma}\|U\|_{H^{\sigma}(-1,1)}, \quad \sigma \geq 1 .
\end{aligned}
$$

Then, (2.12) is easily obtained by the previous theorem, by (2.9), (2.10), and by the triangle inequality:

$$
\|U-v\|_{L^{2}(-1,1)} \leq\left\|U-\Pi_{N} U\right\|_{L^{2}(-1,1)}+\left\|\Pi_{N} U-v\right\|_{L^{2}(-1,1)} .
$$

To show (2.13), it is sufficient to observe that, by definition, $\Pi_{N} U$ coincides with $U$ at $x= \pm 1$ for any $t$, so that

$$
\int_{0}^{T}\left(\Pi_{N} U-U\right)^{2}( \pm 1, t) d t=0, \quad 0<t<T,
$$

and then use the estimates shown above.

Note that the same results hold when $a<0$ and the role of the boundary points $x=1$ and $x=-1$ is interchanged.

\section{ERROR ANALYSIS FOR DIAGONAL SYSTEMS OF EQUATIONS}

Consider the hyperbolic system

$$
\left\{\begin{array}{l}
U_{t}^{(1)}=-A^{(1)} U_{x}^{(1)}, \\
U_{t}^{(2)}=A^{(2)} U_{x}^{(2)},
\end{array} \quad x \in[-1,1], 0<t<T,\right.
$$

where $U^{(1)}(x, t)$ is a vector function of $k_{1} \in \mathbf{N}$ components and $U^{(2)}(x, t)$ is a vector function of $k_{2} \in \mathbf{N}$ components, $\forall x \in[-1,1], \forall t \in[0, T], T>0$. In (3.1), $A^{(1)}$ is a $k_{1} \times k_{1}$ constant matrix and $A^{(2)}$ is a $k_{2} \times k_{2}$ constant matrix. Since (3.1) is a hyperbolic system, we can assume that $A^{(1)}$ and $A^{(2)}$ are positive diagonal matrices.

System (3.1) is subject to initial conditions

$$
\left\{\begin{array}{l}
U^{(1)}(x, 0)=f_{1}(x), \\
U^{(2)}(x, 0)=f_{2}(x),
\end{array} \quad x \in[-1,1],\right.
$$


and it is coupled through the boundary conditions

$$
\left\{\begin{array}{l}
U^{(1)}(-1, t)=L U^{(2)}(-1, t)+g_{1}(t), \\
U^{(2)}(1, t)=R U^{(1)}(1, t)+g_{2}(t),
\end{array} \quad 0<t<T,\right.
$$

where $f_{i}, g_{i}(i=1,2)$ are given functions. Here, $R$ and $L$ are respectively $k_{2} \times k_{1}$ and $k_{1} \times k_{2}$ matrices, which determine the reflection of the ingoing characteristic variables in terms of the outgoing ones at the boundaries.

Let $r$ and $l$ be the norms of the operators $R$ and $L$ respectively, i.e.,

$$
r=\|R\|_{\mathscr{L}\left(\mathbf{R}^{k_{1}}, \mathbf{R}^{\left.k_{2}\right)}\right.}, \quad l=\|L\|_{\mathscr{L}\left(\mathbf{R}^{k_{2}}, \mathbf{R}^{k_{1}}\right)} .
$$

We discuss the case in which the solution to (3.1), (3.2), (3.3) does not grow in time. This is assured by demanding that

$$
0<r l<1 \text {. }
$$

In the new collocation method we seek $u^{(i)}(x, t) \in \mathbf{R}^{k_{i}}, x \in[-1,1], t \in$ $[0, T], i=1,2$. The vectors $u^{(i)}, i=1,2$, whose components are $\mathbf{P}_{N^{-}}$ polynomials in the variable $x$, are determined by the following collocation scheme:

$$
\begin{gathered}
\left\{\begin{array}{l}
u_{t}^{(1)}=-A^{(1)} u_{x}^{(1)} \text { at } x=x_{j}, j=0, \ldots, N-1, \\
u_{t}^{(2)}=A^{(2)} u_{x}^{(2)} \quad \text { at } x=x_{j}, j=1, \ldots, N,
\end{array}\right. \\
\left\{\begin{array}{c}
u_{t}^{(1)}(-1, t)=-A^{(1)} u_{x}^{(1)}(-1, t) \\
-\Gamma^{(1)}\left\{u^{(1)}(-1, t)-L u^{(2)}(-1, t)-g_{1}(t)\right\}, \\
u_{t}^{(2)}(1, t)=A^{(2)} u_{x}^{(2)}(1, t)-\Gamma^{(2)}\left\{u^{(2)}(1, t)-R u^{(1)}(1, t)-g_{2}(t)\right\},
\end{array}\right. \\
\begin{cases}u^{(1)}\left(x_{j}, 0\right)=f_{1}\left(x_{j}\right), & j=0, \ldots, N, \\
u^{(2)}\left(x_{j}, 0\right)=f_{2}\left(x_{j}\right), & j=0, \ldots, N .\end{cases}
\end{gathered}
$$

Here $\Gamma^{(i)}, i=1,2$, are positive-definite $k_{i} \times k_{i}$ diagonal matrices, which can be viewed as amplitudes of the boundary penalty terms, analogous to the role of $\gamma$ in the scalar case. These matrices will be specified later. Note that we took into account at the points $x_{0}=1$ and $x_{N}=1$ both the equations and the boundary conditions.

In order to study the convergence of the $\mathbf{P}_{N}$-approximations $u^{(1)}$ and $u^{(2)}$ to the exact solution $U^{(1)}$ and $U^{(2)}$ when $N$ tends to $+\infty$, we introduce an auxiliary collocation scheme. Namely, we look for $v^{(1)}$ and $v^{(2)}$, such that

$$
\begin{gathered}
\left\{\begin{array}{c}
v_{t}^{(1)}=-A^{(1)} v_{x}^{(1)} \text { at } x=x_{j}, j=0, \ldots, N-1, \\
v_{t}^{(2)}=A^{(2)} v_{x}^{(2)} \text { at } x=x_{j}, j=1, \ldots, N,
\end{array}\right. \\
\left\{\begin{array}{l}
v_{t}^{(1)}(-1, t)=-A^{(1)} v_{x}^{(1)}(-1, t)-\Gamma^{(1)}\left\{v^{(1)}(-1, t)-U^{(1)}(-1, t)\right\} \\
v_{t}^{(2)}(1, t)=A^{(2)} v_{x}^{(2)}(1, t)-\Gamma^{(2)}\left\{v^{(2)}(1, t)-U^{(2)}(1, t)\right\},
\end{array}\right.
\end{gathered}
$$




$$
\begin{cases}v^{(1)}\left(x_{j}, 0\right)=f_{1}\left(x_{j}\right), & j=0, \ldots, N, \\ v^{(2)}\left(x_{j}, 0\right)=f_{2}\left(x_{j}\right), & j=0, \ldots, N .\end{cases}
$$

The advantage of considering (3.9), (3.10), (3.11) is that the new problem is fully decoupled into $k_{1}+k_{2}$ independent scalar equations. Therefore, the analysis of $\S 2$ will enable us to estimate the difference $U^{(i)}-v^{(i)}, i=1,2$. Thus, it remains to bound the difference $u^{(i)}-v^{(i)}, i=1,2$, due to the coupling at the boundaries.

Theorem 3.1. Let $d^{(i)}=u^{(i)}-v^{(i)}, i=1,2$, where $u^{(i)}$ and $v^{(i)}$ are the solutions of (3.6)-(3.8) and (3.9)-(3.11), respectively. Let $\Gamma^{(i)}=A^{(i)} / \omega \sqrt{r l}$, where $\omega=\omega_{0}=\omega_{N}$. Then we have the estimate

$$
\begin{aligned}
\sum_{j=0}^{N}\left(r\left\|B^{(1)} d^{(1)}\left(x_{j}, T\right)\right\|^{2}\right. & \left.+l\left\|B^{(2)} d^{(2)}\left(x_{j}, T\right)\right\|^{2}\right) \omega_{j} \\
\leq \frac{r l}{2(1-\sqrt{r})} \int_{0}^{T} & \left(\sqrt{\frac{r}{l}}\left\|\left(U^{(1)}-v^{(1)}\right)(1, t)\right\|^{2}\right. \\
& \left.+\sqrt{\frac{l}{r}}\left\|\left(U^{(2)}-v^{(2)}\right)(-1, t)\right\|^{2}\right) d t,
\end{aligned}
$$

where $B^{(i)}=\sqrt{\left[A^{(i)}\right]^{-1}}, i=1,2$.

Proof. From (3.6)-(3.11) it is clear that the $d^{(i)}$ satisfy the equations

$$
\begin{cases}d_{t}^{(1)}=-A^{(1)} d_{x}^{(1)} & \text { at } x=x_{j}, j=0, \ldots, N-1, \\ d_{t}^{(2)}=A^{(2)} d_{x}^{(2)} & \text { at } x=x_{j}, j=1, \ldots, N,\end{cases}
$$

$$
\begin{aligned}
& \left\{\begin{aligned}
d_{t}^{(1)}(-1, t)= & -A^{(1)} d_{x}^{(1)}(-1, t) \\
& -\Gamma^{(1)}\left\{d^{(1)}(-1, t)-L d^{(2)}(-1, t)+L E^{(2)}(-1, t)\right\} \\
d_{t}^{(2)}(1, t)= & A^{(2)} d_{x}^{(2)}(1, t) \\
& -\Gamma^{(2)}\left\{d^{(2)}(1, t)-R d^{(1)}(1, t)+R E^{(1)}(1, t)\right\}
\end{aligned}\right. \\
& \begin{cases}d^{(1)}\left(x_{j}, 0\right)=0, & j=0, \ldots, N, \\
d^{(2)}\left(x_{j}, 0\right)=0, & j=0, \ldots, N,\end{cases}
\end{aligned}
$$

where $E^{(i)}=U^{(i)}-v^{(i)}, i=1,2$. The scalar analysis provides us with estimates of the errors $E^{(i)}, i=1,2$. Note that (3.3) has been used to eliminate $U^{(1)}(-1, t)$ and $U^{(2)}(1, t)$.

Now, let $D^{(i)}, i=1,2$, be two diagonal positive-definite $k_{i} \times k_{i}$ matrices to be specified later. Multiplying the first set of equations in (3.13) and (3.14) 
by $\left(D^{(1)} d^{(1)}\right)\left(x_{j}, t\right) \omega_{j}$ and summing over $j=0, \ldots, N$, we get by $(2.2)$

$$
\begin{aligned}
\sum_{j=0}^{N}\left\langle d_{t}^{(1)}\right. & \left., D^{(1)} d^{(1)}\right\rangle\left(x_{j}, t\right) \omega_{j} \\
= & -\int_{-1}^{1}\left\langle A^{(1)} d_{x}^{(1)}, D^{(1)} d^{(1)}\right\rangle(x, t) d x \\
& -\omega\left\langle\Gamma^{(1)} d^{(1)}-\Gamma^{(1)} L d^{(2)}, D^{(1)} d^{(1)}\right\rangle(-1, t) \\
& -\omega\left\langle\Gamma^{(1)} L E^{(2)}, D^{(1)} d^{(1)}\right\rangle(-1, t) \\
\leq & -\frac{1}{2}\left\langle A^{(1)} d^{(1)}, D^{(1)} d^{(1)}\right\rangle(1, t)+\frac{1}{2}\left\langle A^{(1)} d^{(1)}, D^{(1)} d^{(1)}\right\rangle(-1, t) \\
& -\omega\left\langle\Gamma^{(1)} d^{(1)}-\Gamma^{(1)} L d^{(2)}, D^{(1)} d^{(1)}\right\rangle(-1, t) \\
& +\frac{\eta}{2} \omega\left\langle\Gamma^{(1)} d^{(1)}, D^{(1)} d^{(1)}\right\rangle(-1, t)+\frac{\omega}{2 \eta}\left\|\sqrt{D^{(1)} \Gamma^{(1)}} L E^{(2)}(-1, t)\right\|^{2},
\end{aligned}
$$

for any $0<\eta<2$. Similarly, we have

$$
\begin{aligned}
\sum_{j=0}^{N} & \left\langle d_{t}^{(2)}, D^{(2)} d^{(2)}\right\rangle\left(x_{j}, t\right) \omega_{j} \\
& \leq \frac{1}{2}\left\langle A^{(2)} d^{(2)}, D^{(2)} d^{(2)}\right\rangle(1, t)-\frac{1}{2}\left\langle A^{(2)} d^{(2)}, D^{(2)} d^{(2)}\right\rangle(-1, t) \\
& -\omega\left\langle\Gamma^{(2)} d^{(2)}-\Gamma^{(2)} R d^{(1)}, D^{(2)} d^{(2)}\right\rangle(1, t) \\
& +\frac{\eta}{2} \omega\left\langle\Gamma^{(2)} d^{(2)}, D^{(2)} d^{(2)}\right\rangle(1, t)+\frac{\omega}{2 \eta}\left\|\sqrt{D^{(2)} \Gamma^{(2)}} R E^{(1)}(1, t)\right\|^{2}
\end{aligned}
$$

Note that $\eta, D^{(1)}$, and $D^{(2)}$ are yet to be specified. We start by setting $\eta=$ $2(1-\sqrt{r l})$. Recalling the hypotheses on $\Gamma^{(i)}$, by adding (3.16) and (3.17), one obtains

$$
\begin{gathered}
\frac{d}{d t}\left(\sum_{j=0}^{N}\left[\left\|\sqrt{D^{(1)}} d^{(1)}\right\|^{2}+\left\|\sqrt{D^{(2)}} d^{(2)}\right\|^{2}\right]\left(x_{j}, t\right) \omega_{j}\right) \\
\leq-\left[\left\|\sqrt{A^{(1)} D^{(1)}} d^{(1)}\right\|^{2}-\frac{2}{\sqrt{r l}}\left\langle A^{(2)} R d^{(1)}, D^{(2)} d^{(2)}\right\rangle\right. \\
\left.+\left\|\sqrt{A^{(2)} D^{(2)}} d^{(2)}\right\|^{2}\right](1, t) \\
-\left[\left\|\sqrt{A^{(1)} D^{(1)}} d^{(1)}\right\|^{2}-\frac{2}{\sqrt{r l}}\left\langle A^{(1)} L d^{(2)}, D^{(1)} d^{(1)}\right\rangle\right. \\
\left.+\left\|\sqrt{A^{(2)} D^{(2)}} d^{(2)}\right\|^{2}\right](-1, t) \\
+\frac{1}{2(1-\sqrt{r l}) \sqrt{r l}}\left(\left\|\sqrt{D^{(2)} A^{(2)}} R E^{(1)}(1, t)\right\|^{2}\right. \\
\left.+\left\|\sqrt{D^{(1)} A^{(1)}} L E^{(2)}(-1, t)\right\|^{2}\right) .
\end{gathered}
$$


At this point we want the two first terms in brackets on the right-hand side of (3.18) to be positive. This holds if we choose

$$
D^{(1)}=\left(A^{(1)}\right)^{-1} r^{2}, \quad D^{(2)}=\left(A^{(2)}\right)^{-1} r l .
$$

Indeed, in this case the quantity

$$
r^{2}\left\|d^{(1)}\right\|^{2}-2 \sqrt{r l}\left\langle R d^{(1)}, d^{(2)}\right\rangle+r l\left\|d^{(2)}\right\|^{2}
$$

is positive in view of the straightforward inequality

$$
\left|2 \sqrt{r l}\left\langle R d^{(1)}, d^{(2)}\right\rangle\right| \leq\left\|R d^{(1)}\right\|^{2}+r l\left\|d^{(2)}\right\|^{2} \leq r^{2}\left\|d^{(1)}\right\|^{2}+r l\left\|d^{(2)}\right\|^{2} .
$$

Similar arguments hold for the second term. Thus, we are left with the last term, which allows us to write the following inequality:

$$
\begin{aligned}
& \frac{d}{d t}\left(\sum_{j=0}^{N}\left[r\left\|B^{(1)} d^{(1)}\left(x_{j}, t\right)\right\|^{2}+l\left\|B^{(2)} d^{(2)}\left(x_{j}, t\right)\right\|^{2}\right] \omega_{j}\right) \\
& \quad \leq \frac{1}{2(1-\sqrt{r l}) \sqrt{r l}}\left(l\left\|R E^{(1)}(1, t)\right\|^{2}+r\left\|L E^{(2)}(-1, t)\right\|^{2}\right) \\
& \quad \leq \frac{r l}{2(1-\sqrt{r l})}\left(\sqrt{\frac{r}{l}}\left\|E^{(1)}(1, t)\right\|^{2}+\sqrt{\frac{l}{r}}\left\|E^{(2)}(-1, t)\right\|^{2}\right) .
\end{aligned}
$$

Finally, by integrating in time, we obtain (3.12).

Using the results of the previous section, we can finally prove our main convergence theorem.

Theorem 3.2. Let $U^{(i)}, i=1,2$, be the solutions of (3.1), (3.2), (3.3), and let $u^{(i)}, i=1,2$, be the solutions of (3.6), (3.7), (3.8) with

$$
\Gamma^{(i)}=A^{(i)} N(N+1) / 2 \sqrt{r l} .
$$

Then we have the following error estimate:

$$
\begin{aligned}
& \left\|\left(\sum_{i=1}^{2}\left\|U^{(i)}-u^{(i)}\right\|^{2}\right)^{1 / 2}(\cdot, T)\right\|_{L^{2}(-1,1)} \\
& \quad \leq C N^{1-\sigma} T\left\|\left(\sum_{i=1}^{2}\left\|U^{(i)}\right\|^{2}\right)^{1 / 2}\right\|_{C^{0}\left([0, T] ; H^{\sigma}(-1, t)\right)},
\end{aligned}
$$

where $C$ depends only on $l, r, \sigma$, and $A^{(i)}, i=1,2$. 
Proof. We first write $U^{(i)}-u^{(i)}=U^{(i)}-v^{(i)}-d^{(i)}$. Then a bound for each component $U^{(i)}-v^{(i)}, i=1,2$, is given by (2.12), while for $d^{(i)}$ we have

$$
\begin{aligned}
& \left\|\left(\sum_{i=1}^{2}\left\|d^{(i)}\right\|^{2}\right)^{1 / 2}(\cdot, T)\right\|_{L^{2}(-1,1)} \\
& =\left\|\left(\sum_{i=1}^{2}\left\|\left[B^{(i)}\right]^{-1} B^{(i)} d^{(i)}\right\|^{2}\right)^{1 / 2}(\cdot, T)\right\|_{L^{2}(-1,1)}^{2} \\
& \leq \max _{i=1,2}\left\{\left\|\left[B^{(i)}\right]^{-1}\right\|_{\mathscr{L}\left(\mathbf{R}^{k_{i}}, \mathbf{R}^{\left.k_{i}\right)}\right\}}\right\}\left\|\left(\sum_{i=1}^{2}\left\|B^{(i)} d^{(i)}\right\|^{2}\right)^{1 / 2}(\cdot, T)\right\|_{L^{2}(-1,1)}^{2} \\
& \leq C(r, l) \max _{i=1,2} \max _{1 \leq j \leq k_{i}}\left\{\left(a_{j}^{(i)}\right)^{2}\right\}\left(\int_{0}^{T}\left\|U^{(1)}-v^{(1)}\right\|^{2}(1, t) d t\right. \\
& \left.+\int_{0}^{T}\left\|U^{(2)}-v^{(2)}\right\|^{2}(-1, t) d t\right),
\end{aligned}
$$

where $a_{j}^{(i)}$ are the diagonal entries of $A^{(i)}, i=1,2$, and we used Theorem 3.1. Finally, the last term in (3.21) is estimated as in (2.13).

\section{SUGgESTIONS FOR THE IMPLEMENTATION OF NONDIAGONAL SYSTEMS}

In this section we discuss the implementation of our new approach in the case of the general hyperbolic system

$$
\widetilde{U}_{t}=\tilde{A} \widetilde{U}_{x},
$$

where $\widetilde{U} \equiv \widetilde{U}(x, t)$ is a $k$-component vector and $\tilde{A}$ is a constant $k \times k$ matrix with $k_{1}$ negative eigenvalues and $k_{2}$ positive eigenvalues $\left(k=k_{1}+k_{2}\right)$. The following boundary conditions are imposed at $x=-1$ :

$$
\left(\begin{array}{cc}
B_{11} & B_{12} \\
0 & 0
\end{array}\right) \widetilde{U}(-1, t)=\left(\begin{array}{c}
h_{1}(t) \\
0
\end{array}\right)
$$

where $B_{11}$ is a $k_{1} \times k_{1}$ matrix, $B_{12}$ is a $k_{1} \times k_{2}$ matrix, and $h_{1}$ is a given $k_{1}$ component vector. In addition, the following boundary conditions are imposed at $x=1$ :

$$
\left(\begin{array}{cc}
0 & 0 \\
B_{21} & B_{22}
\end{array}\right) \widetilde{U}(1, t)=\left(\begin{array}{c}
0 \\
h_{2}(t)
\end{array}\right)
$$

where $B_{21}$ is a $k_{2} \times k_{1}$ matrix, $B_{22}$ is a $k_{2} \times k_{2}$ matrix, and $h_{2}$ is a given $k_{2}$-component vector. 
By hyperbolicity, there exists a nonsingular matrix

$$
T=\left(\begin{array}{ll}
T_{11} & T_{12} \\
T_{21} & T_{22}
\end{array}\right)
$$

where $T_{i j}$ is a $k_{i} \times k_{j}$ matrix, such that the change of variables $\tilde{U}=T U$ diagonalizes the system (4.1). Thus, we get

$$
\frac{\partial U}{\partial t}=T^{-1} \tilde{A} T \frac{\partial U}{\partial x}=\left(\begin{array}{cc}
-A^{(1)} & 0 \\
0 & A^{(2)}
\end{array}\right) \frac{\partial U}{\partial x},
$$

where $U \equiv\left(U^{(1)}, U^{(2)}\right)$ and the $A^{(i)}, i=1,2$, have been defined in $\S 3$.

The boundary conditions are respectively transformed as follows:

$$
\begin{gathered}
\left(B_{11} T_{11}+B_{12} T_{21}\right) U^{(1)}(-1, t)+\left(B_{11} T_{12}+B_{12} T_{22}\right) U^{(2)}(-1, t)=h_{1}(t) \\
\left(B_{21} T_{11}+B_{22} T_{21}\right) U^{(1)}(1, t)+\left(B_{21} T_{12}+B_{22} T_{22}\right) U^{(2)}(1, t)=h_{2}(t) .
\end{gathered}
$$

Therefore, (4.2) and (4.3) are equivalent to (3.3) if and only if the matrices $B_{11} T_{11}+B_{12} T_{21}$ and $B_{21} T_{12}+B_{22} T_{22}$ are invertible. In this case we have:

$$
\begin{gathered}
L=-\left(B_{11} T_{11}+B_{12} T_{21}\right)^{-1}\left(B_{11} T_{12}+B_{12} T_{22}\right), \\
R=-\left(B_{21} T_{12}+B_{22} T_{22}\right)^{-1}\left(B_{21} T_{11}+B_{22} T_{21}\right), \\
g_{1}=\left(B_{11} T_{11}+B_{12} T_{21}\right)^{-1} h_{1}, \quad g_{2}=\left(B_{21} T_{12}+B_{22} T_{22}\right)^{-1} h_{2} .
\end{gathered}
$$

Thus, assumption (3.5) is transferred to the $B_{i j}$.

We would like to show how to apply the scheme (3.9)-(3.11) directly to the system (4.1)-(4.3). According to Theorem 3.2 we set $\Gamma^{(i)}=\beta A^{(i)}, i=1,2$, where $\beta=N(N+1) / 2 \sqrt{r l}$. We also set (denoting by $u \equiv\left(u^{(1)}, u^{(2)}\right)$ the approximation to $U$ )

$$
\begin{aligned}
\Xi(x, t) & =\left(\begin{array}{c}
u^{(1)}(x, t)-L u^{(2)}(x, t)-g_{1}(t) \\
u^{(2)}(x, t)-R u^{(1)}(x, t)-g_{2}(t)
\end{array}\right) \\
& =\left(\begin{array}{cc}
I & -L \\
-R & I
\end{array}\right) u(x, t)-\left(\begin{array}{l}
g_{1} \\
g_{2}
\end{array}\right)(t) .
\end{aligned}
$$

Thus, (3.1)-(3.3) can be written in the form

$$
\begin{aligned}
\frac{\partial u}{\partial t}\left(x_{j}, t\right)= & A \frac{\partial u}{\partial x}\left(x_{j}, t\right)+\beta \delta_{N j} A^{(-)} \Xi(-1, t) \\
& -\beta \delta_{0 j} A^{(+)} \Xi(1, t), \quad j=0, \ldots, N,
\end{aligned}
$$

where

$$
A^{(-)}=\left(\begin{array}{cc}
-A^{(1)} & 0 \\
0 & 0
\end{array}\right), \quad A^{(+)}=\left(\begin{array}{cc}
0 & 0 \\
0 & A^{(2)}
\end{array}\right), \quad A=A^{(-)}+A^{(+)},
$$

and $\delta_{i j}$ is the Kronecker delta. 
Let us now define $\tilde{u}=T u$. The function $\tilde{u}$ satisfies

$$
\begin{aligned}
\frac{\partial \tilde{u}}{\partial t}\left(x_{j}, t\right)= & \tilde{A} \frac{\partial \tilde{u}}{\partial x}\left(x_{j}, t\right)+\beta \delta_{N j}\left(T A^{(-)} T^{-1}\right) T \Xi(-1, t) \\
& -\beta \delta_{0 j}\left(T A^{(+)} T^{-1}\right) T \Xi(1, t), \quad j=0, \ldots, N .
\end{aligned}
$$

Defining $B=T\left(\begin{array}{cc}I & -L \\ -R & I\end{array}\right) T^{-1}$, we get

$$
T \Xi=B \tilde{u}-T\left(\begin{array}{l}
g_{1} \\
g_{2}
\end{array}\right) \text {. }
$$

Finally, taking $\widetilde{A}^{ \pm)}=T A^{( \pm)} T^{-1}$ and substituting in (4.8), we obtain the pseudospectral scheme for approximating (4.1)-(4.3), namely

$$
\begin{aligned}
\frac{\partial \tilde{u}}{\partial t}\left(x_{j}, t\right)= & \tilde{A} \frac{\partial \tilde{u}}{\partial x}\left(x_{j}, t\right)+\beta \delta_{N j} \tilde{A}^{-)}\left[B \tilde{u}(-1, t)-T\left(\begin{array}{l}
g_{1} \\
g_{2}
\end{array}\right)(t)\right] \\
& -\beta \delta_{0 j} \widetilde{A}^{(+)}\left[B \tilde{u}(1, t)-T\left(\begin{array}{l}
g_{1} \\
g_{2}
\end{array}\right)(t)\right], \quad j=0, \ldots, N .
\end{aligned}
$$

This is equivalent to collocating the equation (4.1) at all the points with some suitable penalty terms, derived from the boundary conditions, added at the points $x= \pm 1$. It is clear that the same convergence estimates of Theorem 3.2 also apply for the error $\widetilde{U}-\tilde{u}=T(U-u)$.

\section{BIBLIOGRAPHY}

1. C. Canuto, M. Y. Hussaini, A. Quarteroni, and T. A. Zhang, Spectral methods in fluid dynamics, Springer, New York, 1988.

2. P. J. Davis and P. Rabinowitz, Methods of numerical integration, 2nd ed., Academic Press, New York, 1984.

3. D. Funaro and D. Gottlieb, A new method of imposing boundary conditions in pseudospectral approximations of hyperbolic equations, Math. Comp. 51 (1988), 599-613.

4. D. Gottlieb, L. Lustman, and E. Tadmor, Stability analysis of spectral methods for hyperbolic initial-boundary value systems, SIAM J. Numer. Anal. 24 (1987), 241-256.

5. __ Convergence of spectral methods for hyperbolic initial-boundary value systems, SIAM J. Numer. Anal. 24 (1987), 532-537.

6. B. Gustafsson, H. O. Kreiss, and A. Sundström, Stability theory of difference approximations for mixed initial boundary value problems. II, Math. Comp. 26 (1972), 649-686.

7. S. Osher, Stability of difference approximations of dissipative type for mixed initial-boundary value problems. I, Math. Comp. 23 (1969), 335-340.

Dipartimento di Matematica, Università di Pavia, Strada Nuova 65, 27100 Pavia, Italy

Division of Applied Mathematics, Brown University, Providence, Rhode IsLand 02912 